XXVII International Conference on Photonic, Electronic and Atomic Collisions (ICPEAC 2011) IOP Publishing Journal of Physics: Conference Series 388 (2012) $022053 \quad$ doi:10.1088/1742-6596/388/2/022053

\title{
Differential cross-sections for the double photoionization of lithium
}

\author{
A. S. Kheifets ${ }^{* 1}$, D. V. Fursa ${ }^{\star}$ Igor Bray ${ }^{\star}$, J. Colgan ${ }^{\dagger}$ and M. S. Pindzola ${ }^{\ddagger}$ \\ *Research School of Physics, The Australian National University, Canberra ACT 0200, Australia \\ *ARC Centre for Matter-Antimatter Studies, Curtin University, WA 6845 \\ ${ }^{\dagger}$ Theoretical Division, Los Alamos National Laboratory, Los Alamos, NM 87545, USA \\ ${ }^{\ddagger}$ Department of Physics, Auburn University, Auburn, AL 36849, USA
}

Synopsis We apply the convergent close-coupling (CCC) and time-dependent close- coupling (TDCC) methods to describe energy and angular resolved double photoionization (DPI) of lithium at arbitrary energy sharing. By doing so, we are able to evaluate the recoil ion momentum distribution of DPI of Li and make a comparison with recent measurements of Zhu et al. [Phys. Rev. Lett. 103, 103008 (2009)].

The lithium atom is the simplest target, beyond $\mathrm{He}$ and $\mathrm{H}_{2}$, for which differential, with respect to the photoelectron energies and angles (or momenta), characterization of double photoionization (DPI) is now possible. Zhu et al. [1] performed differential measurements of DPI of $\mathrm{Li}$ resolved with respect to the sum photoelectron momentum (or, equivalently, the recoil ion momentum). On the theory side, the convergent close-coupling (CCC) and time-dependent closecoupling (TDCC) methods are now capable of producing fully-resolved triply-differential crosssections (TDCS) for $\mathrm{Li}[2]$

In the special case of equal energy sharing condition, the TDCS in lithium can be conveniently parametrized by a pair of symmetrized DPI amplitudes in the singlet and triplet channels. The partial contribution of these amplitudes varies with the fixed escape angle relative to the polarization axis of light. The angular spread of the amplitudes relative to the back-toback emission indicates the strength of the angular correlation in the two-electron continuum. This strength depends noticeably on the spin of the photoelectron pair [3].

In the general case of arbitrary energy sharing, the number of symmetrized DPI amplitudes should be doubled. To describe the experimental recoil ion momentum distribution [1], a complete set of TDCS should be integrated across various energy sharings and mutual photoelectron angles. This distribution is given by the following differential cross-section:

$$
\frac{d^{2} \sigma}{d p d \Omega_{p}}=\frac{1}{4 \pi} \sum_{S=0,1} \frac{d \sigma_{S}}{d p}\left[1+\beta_{S} P_{2}\left(\cos \theta_{p}\right)\right]
$$

where the asymmetry parameter $\beta$ and the single differential, with respect to the momentum, cross-section (SDCS) are expressed via the angular integrals of the amplitudes in the singlet

\footnotetext{
${ }^{1}$ E-mail: A.Kheifets@anu.edu.au
}

$S=0$ and triplet $S=1$ channels. Similarly, one can obtain the double differential crosssection (DDCS) $d^{2} \sigma / d p_{x} d p_{z}$ and compare it directly with experiment (see Fig. 1).

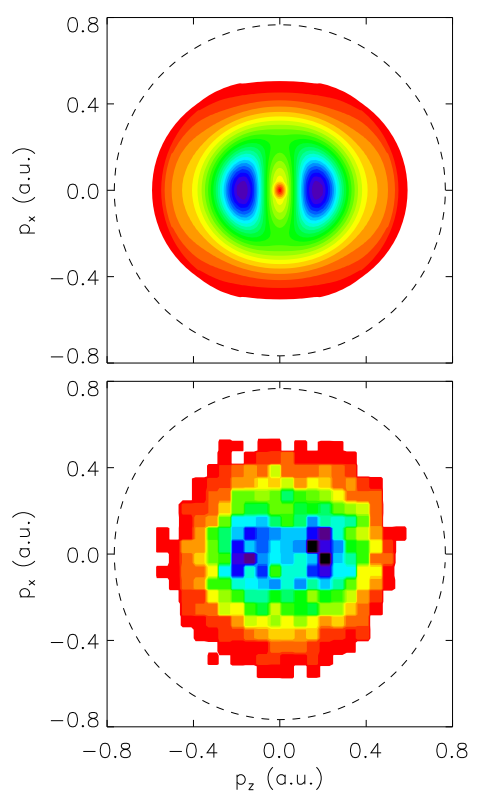

Figure 1. The DDCS $d^{2} \sigma / d p_{x} d p_{z}$ of DPI of $\mathrm{Li}$ at the photon energy of $\omega=85 \mathrm{eV}$. Top: theory [2], bottom: experiment [1]. The dashed circle on each panel indicates the maximum available recoil momentum $p_{\max }=0.77$ a.u.

\section{References}

[1] G. Zhu et al 2009 Phys. Rev. Lett. 103(10):103008

[2] A. S. Kheifets et al 2010 Phys. Rev. A 82(2):023403

[3] A. S. Kheifets et al 2010 Phys. Rev. A 81(2):023418 